\title{
Thioridazine induces apoptosis by targeting the PI3K/Akt/mTOR pathway in cervical and endometrial cancer cells
}

\author{
Sokbom Kang • Seung Myung Dong • \\ Boh-Ram Kim • Mi Sun Park • Barry Trink • \\ Hyun-Jung Byun $\cdot$ Seung Bae Rho
}

Published online: 30 March 2012

(C) The Author(s) 2012. This article is published with open access at Springerlink.com

\begin{abstract}
Recently, thioridazine (10-[2-(1-methyl-2piperidyl) ethyl]-2-methylthiophenothiazine), a wellknown anti-psychotic agent was found to have anti-cancer activity in cancer cells. However, the molecular mechanism of the agent in cellular signal pathways has not been well defined. Thioridazine significantly increased earlyand late-stage apoptotic fraction in cervical and endometrial cancer cells, suggesting that suppression of cell growth by thioridazine was due to the induction of apoptosis. Cell cycle analysis indicated thioridazine induced the downregulation of cyclin D1, cyclin $\mathrm{A}$ and $\mathrm{CDK} 4$, and the induction of p21 and p27, a cyclin-dependent kinase inhibitor. Additionally, we compared the influence of thioridazine with cisplatin used as a control, and similar patterns between the two drugs were observed in cervical
\end{abstract}

Sokbom Kang, Seung Myung Dong contributed equally to this work.

Electronic supplementary material The online version of this article (doi:10.1007/s10495-012-0717-2) contains supplementary material, which is available to authorized users.

S. Kang - S. M. Dong - B.-R. Kim - M. S. Park - H.-J. Byun · S. B. Rho ( $\square)$

Research Institute, National Cancer Center, 323, Ilsan-ro, Ilsandong-gu, Goyang-si, Gyeonggi-do 410-769,

Republic of Korea

e-mail: sbrho@ncc.re.kr

\section{S. Kang}

Division of Gynecologic Cancer Research, Research Institute and Hospital, National Cancer Center, 323, Ilsan-ro, Ilsandonggu, Goyang-si, Gyeonggi-do 410-769, Republic of Korea

B. Trink

Division of Head and Neck Cancer Research, Department of Otolaryngology and Head \& Neck Surgery, The Johns Hopkins University School of Medicine, Baltimore, MD 21231, USA and endometrial cancer cell lines. Furthermore, as expected, thioridazine successfully inhibited phosphorylation of Akt, phosphorylation of 4E-BP1 and phosphorylation of p70S6K, which is one of the best characterized targets of the mTOR complex cascade. These results suggest that thioridazine effectively suppresses tumor growth activity by targeting the PI3K/Akt/mTOR/p70S6K signaling pathway.

Keywords Thioridazine - Apoptosis - Anti-cancer activity $\cdot$ mTOR signaling $\cdot$ Cervical tumorigenesis
Abbreviations
Thioridazine
10-[2-(1-methyl-2-piperidyl) ethyl]-2- methylthiophenothiazine
mTOR The mammalian target of rapamycin
CDK Cyclin dependent kinase
MTT
3-(4,5-dimethylthiazol-2-yl)-2.5-
FITC diphenyl- ${ }^{2} \mathrm{H}$-tetrazolium bromide
PI Fluorescein isothiocyanate
Propidium iodide

\section{Introduction}

Thioridazine (10-[2-(1-methyl-2-piperidyl) ethyl]-2-methylthiophenothiazine), a phenothiazine derivative, is a potent anti-psychotic and anti-anxiety agent belonging to the phenothiazine drug family. It is widely used to treat psychotic disorders such as psychosis and schizophrenia. Thioridazine may interact with other drugs which can cause drowsiness, such as alcohol, anti-depressants, muscle relaxants, anti-histamines, pain relievers and anxiety medicines. In case of advanced cancer, the drug has been used to treat cancerrelated sweating $[1,2]$ and depression [3]. However, there have been several previous reports showing that the drug may 
cause serious side effects and a reversal of drug resistance, as well as, neuroleptic malignant syndrome [4-8]. Although several mechanisms, including inhibition of P-glycoprotein [9], DNA damage [10], or anti-oxidant activity [11], have been proposed to explain the anti-cancer effects of the drug, the true mechanism of thioridazine's anti-cancer effects have been unclear.

Recently, a group of researchers observed that the antiproliferative effect of chlorpromazine, another phenothiazine derivative, can be blocked by wortmannin, a selective PI3K inhibitor [12]. Thioridazine use may lead to the development of symptoms that resemble Parkinson's disease, but these symptoms are not directly caused by Parkinson's. In a study, we demonstrated the similiarities of the gene expression profile between thioridazine and known PI3K/Akt pathway inhibitors using the geneexpression-based query [13], while also showing that thioridazine could exhibit PI3K/Akt pathway inhibition in ovarian cancer cells.

Phosphatidylinositol 3-kinase (PI3K)/Akt signal transduction plays an important role in cell growth via inhibition of apoptosis in various types of human cancers [14-17]. Activation of Akt also promotes tumor metastasis and invasion, antagonizes cell-cycle arrest, angiogenesis, and phosphorylates mTOR (mammalian target of rapamycin) protein kinase. The mTOR pathway is mediated by a wide variety of cellular signal communications which include hormones, such as insulin and growth factors, nutrients, such as amino acids and glucose, and cellular stress conditions. The phosphorylation of Akt is modulated by phosphatidylinositol-3,4-bisphosphate and phosphatidylinositol-3,4,5-triphosphate, which are generated by (PI3K) [18]. PI3K is an activator of Akt, which consists of catalytic subunits $(110 \mathrm{kDa})$ and regulatory subunits $(85 \mathrm{kDa})$ [19]. A principal pathway that signals via mTOR is the PI3K/Akt signaling pathway, which is critically involved in the regulation of cell proliferation and survival. mTOR may also indirectly influence the phosphorylation condition of 4E-BP1 by modulating the activity of PP2A (Protein Phosphatase-2A). The second step effector, which is down-stream of mTOR, is the p70S6K serine/threonine kinase. After processing a cell proliferative up-stream signal mediated by the PI3K/Akt pathway, mTOR phosphorylates and activates p70S6K. mTOR plays a key role in the regulation of cell cycle progression, which includes protein synthesis, tumor growth, and angiogenesis [20-22].

In the present study, we estimated the anti-proliferative effect of thioridazine in human cervical and endometrial cancer cells, and determined the underlying molecular mechanisms. In addition, here we showed that thioridazine is a potent suppressor of cellular signaling pathways of PI3K-Akt-mTOR.

\section{Materials and methods}

Cell culture, antibodies, and chemicals

Human cervical (HeLa, C33A and Caski) and endometrial (HEC-1-A and KLE) cancer cell lines were maintained in DMEM (Life Technologies, Gaithersburg, MD) and RPMI1640 added with either $10 \%$ heat-inactivated fetal bovine serum (FBS), penicillin (100 U/ml), or streptomycin $(100 \mu \mathrm{g} / \mathrm{ml})$, at $37{ }^{\circ} \mathrm{C}$ in a humidified atmosphere containing $5 \% \mathrm{CO}_{2}$. The following antibodies were used in this study: anti-Akt, anti-phospho-specific Akt, anti-PI3K, anti-phospho-specific PI3K, anti-cyclin A, anti-cyclin B1, anti-cyclin D1, anti-CDK1, anti-CDK2, anti-CDK4 (Santa Cruz Biotechnology, Santa Cruz, CA), anti-caspase-3, antiBcl-2, anti-Bcl-xL, anti-Bax, anti-mTOR, anti-phosphomTOR, anti-p70S6K, and anti-phospho-p70S6K(Thr 421) (BDPhamingen, San Diego, CA), anti-p21 and anti-p27 (Oncogene, San Diego, CA), anti-4E-BP1 and anti-phospho-4E-BP1 (Cell Signaling) and anti- $\beta$-actin (Sigma). Rapamycin was purchased from Cell Signaling (Berverly, MA). Other chemicals and anticancer drugs were purchased from Sigma (St. Louis, MO).

\section{Cell viability assays}

The viability of cell growth was determined using 3-(4,5dimethylthiazol-2-yl)-2.5-diphenyl- ${ }^{2} \mathrm{H}$-tetrazolium bromide (MTT) assays. Briefly, cell lines were grown in DMEM medium containing $10 \%$ FBS. The cells were plated at a density of $3.4 \times 10^{3}$ cells/well in 96-well plates. After $24 \mathrm{~h}$, fresh medium containing $10 \%$ FBS and $20 \mu \mathrm{l}$ of MTT solution (Sigma, $5 \mathrm{mg} / \mathrm{ml}$ ) was added to each well. Each well was then incubated for an additional $4 \mathrm{~h}$ at $37^{\circ} \mathrm{C}$. The amount of MTT-formazan generated was measured as the absorbance by using a microculture plate reader at $540 \mathrm{~nm}$. Each individual measurement was repeated three times.

Cell cycle analysis and apoptosis assays

Cancer cells were distributed onto six-well plates and then treated with thioridazine $(15 \mu \mathrm{M})$ for $24 \mathrm{~h}$, respectively. To evaluate apoptotic cells, the nuclei were fixed in methanol, stained with $2 \mu \mathrm{g} / \mathrm{ml}$ of 4,6'-diamidino-2-phenylindole (DAPI, Boehringer Mannheim, Mannhein, Germany) for $15 \mathrm{~min}$ at $37^{\circ} \mathrm{C}$, rinsed twice with PBS, and monitored under a fluorescence microscope (Zeiss; Switzerland). All experiments were carried out in triplicate. Subsequently, for the analysis of the DNA content using flow cytometry, each cell line was maintained at a density of $3.2 \times 10^{5}-$ $4.1 \times 10^{5}$ cells in $60-\mathrm{mm}$ plates. After treatment with thioridazine, the cells were harvested, rinsed with ice-cold PBS, and fixed with ice-cold $70 \%$ ethanol. The cells were 
centrifuged for $5 \mathrm{~min}$ at $1,000 \times g$ and resuspended in PBS containing $5 \mathrm{mM}$ of EDTA and RNase A $(1 \mathrm{mg} / \mathrm{ml})$. After incubation for $1 \mathrm{~h}$ at $37^{\circ} \mathrm{C}$, the cells were treated for $15 \mathrm{~min}$ with fluorescein isothiocyanate (FITC)-labeled Annexin V and propidium iodide (PI), according to the supplier's protocols (Boehringer Mannheim, Mannhein), and then were analyzed with a flow cytometer (FACScalibur, Becton-Dickinson, Franklin Lakes, NJ).

\section{Measurement of caspase- 3 activity}

For caspase-3 activity, cells $\left(2.5 \times 10^{6}\right)$ were grown in either the absence or presence of thioridazine at $37{ }^{\circ} \mathrm{C}$ for $24 \mathrm{~h}$. Caspase-3 activity was measured using an actyl-DEVD-7-amino-4-trifluoromethyl coumarin as the substrate, according to the manufacturer's instructions (BDPharmingen, San Diego). In brief, the cells were placed with VP-16 $(100 \mu \mathrm{g} / \mathrm{ml})$ for $24 \mathrm{~h}$, lysed in lysis buffer, and centrifuged for $25 \mathrm{~min}$ at $12,000 \times g$ at $4{ }^{\circ} \mathrm{C}$. The activity was quantified in the supernatant fraction according to its proteolytic cleavage of the colorimetric substrate by use of a Spectramax 340 microplate reader (Molecular Devices, Sunnyvale) in fluorescence mode, with excitation at $405 \mathrm{~nm}$ and emission at $505 \mathrm{~nm}$.

For assay of PARP cleavage, we carried out the procedures as described in the previous study [23, 24]. In brief, $50 \mu \mathrm{g}$ of protein was placed with $60 \mu \mathrm{M}$ biotinylated NAD in a $50 \mu \mathrm{l}$ final volume of PARP reaction buffer $(50 \mathrm{mM}$ Tris- $\mathrm{HCl}, \mathrm{pH} 8.0$ and $25 \mathrm{mM} \mathrm{MgCl}_{2}$ ) at $37{ }^{\circ} \mathrm{C}$ for $1 \mathrm{~h}$. The reaction was stopped by the addition of SDS loading dye buffer, and the products were separated using SDS-PAGE gel and autoradiography.

\section{Immunoblotting analysis}

After treatment with thioridazine, cells were harvested by centrifugation. Cell extracts were prepared by washing cells with PBS, and cells were lysed in a buffer containing protease inhibitor. The protein yield was measured using the Bio-Rad protein assay kit. Equal amounts of protein were loaded, separated by SDS-PAGE gel, and then transferred to polyvinylidene difluoride membrane. After blocking, the membranes were placed at room temperature for $1 \mathrm{~h}$ with primary antibodies applied. The blots were washed thrice in wash buffer and incubated with the appropriate horseradish peroxidase-linked secondary antibodies. The immunoreactive bands were developed using the ECL detection system.

\section{Luciferase assays}

Luciferase activity was carried out with a dual luciferase reporter assay kit (Promega, Madison, WI). Cancer cells were transfected by using the vector DNA containing p21-, p53- and Bcl-2-luciferase, in which the luciferase is expressed under each promoter control. The reporter plasmid, Bcl-2-Luc and p53-Luc were kindly provided by Dr. K. Park (Samsung Medical Center, Korea), and p21 promoter reporter construct by J. Park (Yonsei University, Korea). Data presented are representative of three replicate experiments. Briefly, cells at $80 \%$ confluency were transiently transfected with each indicated reporter construct. After lysis with Reporter Lysis Buffer (Promega), lysates were cleared with centrifugation for $15 \mathrm{~min}$ at $14,000 \mathrm{rpm}$ and cell extracts were incubated with the luciferase substrate reagent for $30 \mathrm{~min}$ at room temperature. Then, a $5 \mu \mathrm{l}$ aliquot of each sample was transferred into the MicroLumat Plus LB96V luminometer. The ratio was normalized for the Renilla luciferase activity to correct for variations in transfection efficiency.

\section{PI3K assays}

Enzyme assays were carried out as described previously by Fruman et al. [25]. In brief, cells were plated at a density of $1.8 \times 10^{6}$ cells. After overnight incubation, cells were treated with either $15 \mu \mathrm{M}$ thioridazine or wortmannin and LY294002 as a PI3K inhibitor, or left without treatment as the control for $24 \mathrm{~h}$. Cells were lysed in $1 \%$ NP-40 lysis buffer containing $20 \mathrm{mM}$ Tris- $\mathrm{HCl}$ (pH 7.5), $100 \mathrm{mM} \mathrm{NaCl}, 1 \mathrm{mM}$ EDTA, $1 \mathrm{mM} \mathrm{MgCl}_{2}, 1$ $\%$ NP-40, $1 \mathrm{mM}$ phenylmethylsulfonyl fluoride, and $0.1 \mathrm{mM}$ sodium orthovanadate. The lysates were centrifuged at $4{ }^{\circ} \mathrm{C}$ for $15 \mathrm{~min}$ at $20,000 \times g$ and the supernatants were used as the cell lysate. To immunoprecipitate $\mathrm{PI} 3 \mathrm{~K}$, proteins were incubated for $1 \mathrm{~h}$ at $4{ }^{\circ} \mathrm{C}$ with antip85 antibody, followed by incubation with protein A-agarose beads for an additional $1 \mathrm{~h}$ at $4{ }^{\circ} \mathrm{C}$. Immnunoprecipitates were incubated with kinase reaction buffer mix containing $200 \mu \mathrm{g} / \mathrm{ml}$ of phosphatidylinositol and $2 \mu \mathrm{Ci}$ of $\left[-{ }^{32} \mathrm{P}\right]$ ATP per assay mixture at $37{ }^{\circ} \mathrm{C}$ for $15 \mathrm{~min}$. The reaction products were developed using autoradiography and the radioactive lipids were quantified by liquid scintillation counting.

\section{Statistical analyses}

All data values were presented as either the mean \pm SD (mean \pm standard deviation) or means \pm SEM (means \pm standard error of means). Statistical comparisons were measured using Student's $t$-test. Statistical analyses were carried out by using STATA software ver. 10.0 (StataCorp, College Station, TX). $P$ values of $<0.05(*)$ were considered significant. 


\section{Results}

Thioridazine regulates the cell proliferation and apoptosis

In order to examine the effect of thioridazine-induced inhibition of cellular proliferation in cervical and endometrial cancer cells, cells were treated with thioridazine $(15 \mu \mathrm{M})$. As shown in Fig. 1a, cell viability of the cervical (HeLa, Caski and C33A) and endometrial (HEC-1-A and KLE) cancer cells were reduced by treatment of thioridazine. To confirm that the reduction in the cell numbers was reflective of cell death, flow cytometric detection was performed using labeled annexin $\mathrm{V}$ (Fig. 1b). Among all tested cell lines except KLE cells, cells treated with thioridazine demonstrated significantly increased early- and late-stage apoptotic fraction, which suggests that cell growth suppression by thioridazine was due to increased apoptosis. In addition, we compared the influence of thioridazine with cisplatin and similar apoptotic patterns between the two agents were observed in HeLa cells.

Subsequently, we assessed whether this effect of thioridazine is associated with an activation of caspase-3. In western blot analysis, as well as activity, thioridazine clearly induced activation of caspase-3. Especially in HeLa and HEC-1-A, the degree of caspase-3 activity was comparable to that of cisplatin (Fig. 1c). Additionally, cleavage of caspase- 3 was also confirmed in response to thioridazine in HeLa and HEC-1-A, indicating that caspase-3 activation was mediated by cleavage of caspase-3 (Fig. 1d). We also compared the potency of thioridazine in cell proliferation inhibition with those of wortmannin and LY294002. As shown in Supplementary Fig. 1, proliferation of cells treated with wortmannin or LY294002 was inhibited to 53 and $52 \%$ lower than the control in HeLa cells. In addition, thioridazine had more cell growth inhibitory effect than either of these two known PI3K inhibitors. Finally, the cleavage and activation of caspase- 3 resulted in the characteristic proteolysis, e.g., cleavage of poly (ADP-ribose) polymerase (PARP) of HeLa and HEC-1-A after treatment of thioridazine (Fig. 1e). These results corresponded to our previous observation that thioridazine may inhibit cellular proliferation and induce apoptosis.

Thioridazine-induced alteration of cell cycle modulators

Since we previously found that thioridazine may modulate the regulation of cell cycle progression by interfering with the PI3K/Akt pathway and induces $G_{1}$ cell cycle arrest, we then investigated the influence of thioridazine treatment on changes to the cell cycle regulatory proteins using immunoblotting assays. As expected, we observed that thioridazine dramatically inhibited the expression of cyclin D1 and CDK4. In addition, p27 protein expression was enhanced after treatment of thioridazine, suggesting CDK inhibitor p27 has a major role in $G_{1}$ cell cycle arrest induced by thioridazine. We also observed reduced expression of cyclin A and increased level of p21, which was consistent with a $G_{1}$ cell cycle arrest. The expression of cyclin A and CDK2, which regulates transition from $\mathrm{S}$ or $M$ phase, was decreased with the treatment of thioridazine. CDK1 and cyclin B1 regulate progressions from $G_{2}$ to $M$ phase, and were also inhibited (Fig. 2a).

Since p21 is the transcriptional target of p53, the expression of p53 was explored (Fig 2b). Expression level of p53 was increased after treatment of thioridazine along with Bax. In contrast, expression of anti-apoptotic protein Bcl-2 and Bcl-xL was decreased. Furthermore, by using luciferase reporter assay, we confirmed that thioridazine increased transcription of p53 (Fig. 2c). In the Supplementary Fig. 2, p21 and p27 more enhanced the expression levels of wortmannin-treated HeLa cells when compared with that of thioridazine-treated cells as expected. Also, treatment of thioridazine or wortmannin significantly increased the levels of Bax and p53 expression in a time-dependent manner. Thus, it can be speculated that thioridazine induces $G_{1}$ cell cycle arrest by increasing transcription of p53 and its transcriptional target, p21.

\section{Suppression of PI3K/Akt/mTOR/p70S6K}

phosphorylation by thioridazine in cervical and endometrial cancer cells

We tested whether thioridazine could induce inhibition of PI3K activity in HeLa and HEC-1-A cells. After treatment with EGF (50 ng/ml, $6 \mathrm{~h}$ ), cell lysates were immunoprecipitated using anti-p85 antibody with or without thioridazine treatment. After treatment of thioridazine, cells showed significantly inhibited phosphorylation of PI3K. This inhibitory activity was comparable to that of two wellknown PI3K inhibitor, wortmannin and LY294002 (Fig. 3a). The inhibition of PI3K in the two cells resulted in inhibition of Akt, which is one of the major downstream targets of PI3K. As expected, thioridazine also successfully inhibited phosphorylation of Akt (Ser 473) and phosphorylation of 4E-BP1 (Fig. 3b), one of the best characterized targets of the mTOR complex. Also, treatment with thioridazine decreased the level of phosphorylated Akt (Thr 308 ) as well as the level of phosphorylated GSK-3 $\beta$ (Ser 9) within 3-6 h, whereas wortmannin and LY294002 significantly inhibited the level of phosphorylated protein within $6 \mathrm{~h}$ (Supplementary Fig. 3). When thioridazine was co-treated with rapamycin, inhibitor of mTOR, we observed that they exerted additive effects in the 
A

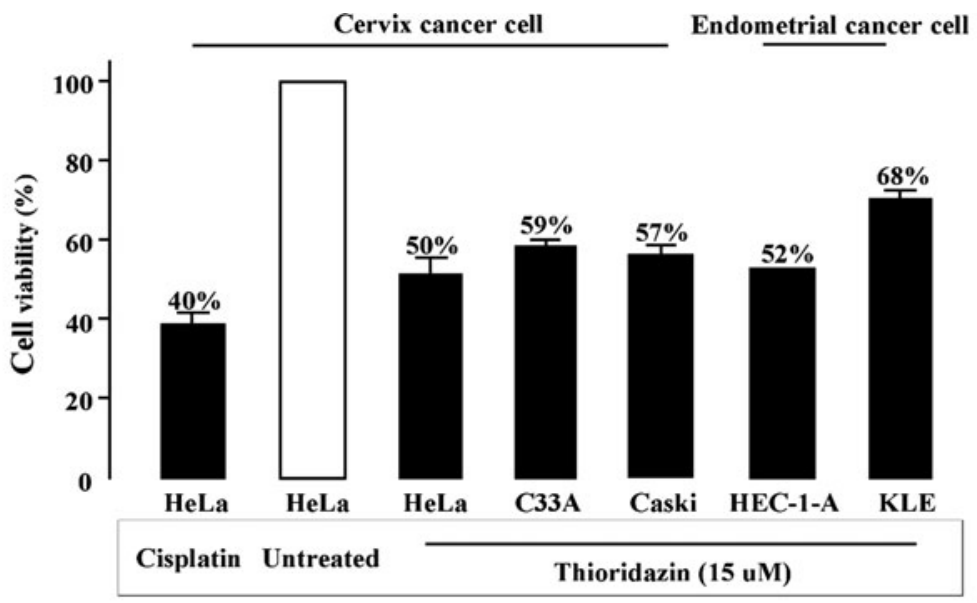

B
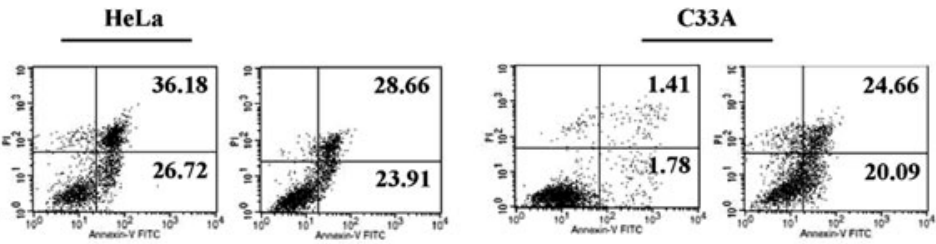

Untreated

Thioridazine
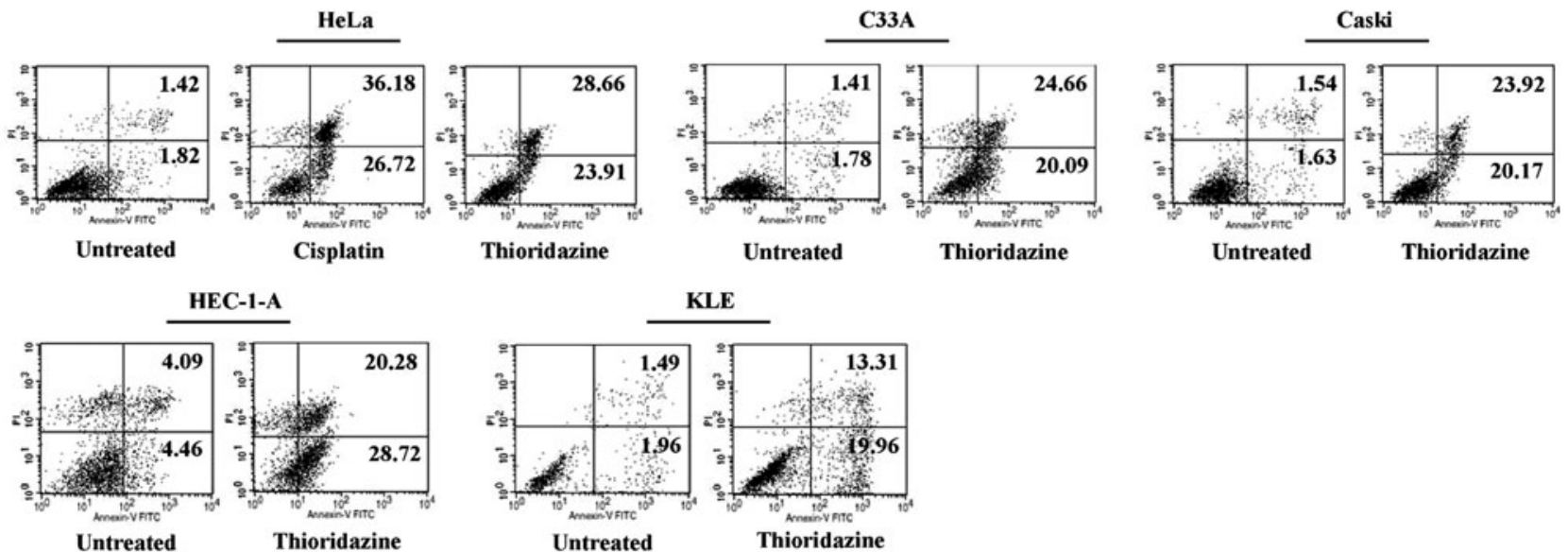

$\mathbf{E}$

D

C

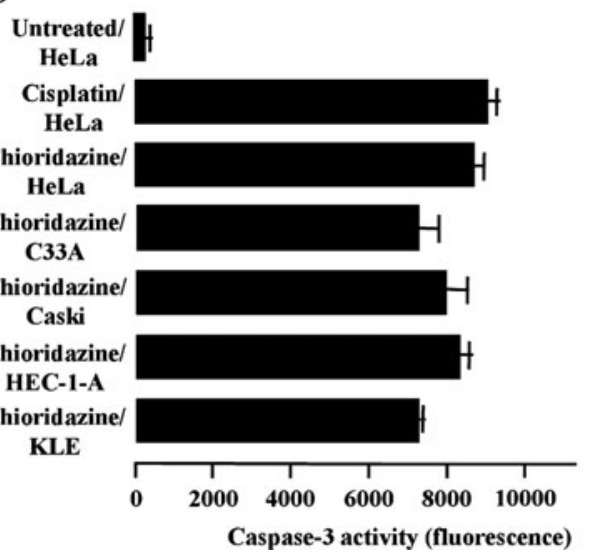

Fig. 1 Treatment of thioridazine inhibits cellular proliferation and activates caspase-dependent pro-apoptotic activity. a Effect of treatment with thioridazine $(15 \mu \mathrm{M})$ was analyzed by 3-(4,5-dimethylthiazol-2-yl)-2.5-diphenyl- ${ }^{2} \mathrm{H}$-tetrazolium bromide (MTT) assays. Inhibition of cellular proliferation by cisplatin $(20 \mu \mathrm{M})$ in HeLa cells was also illustrated for comparison. b Early- and late-stage apoptosis induced by thioridazine was analyzed by fluorescein isothiocyanate

deactivation of $\mathrm{p}-4 \mathrm{E}-\mathrm{BP} 1$. These results suggest that confirm our former hypothesis that thioridazine is a potent inhibitor of PI3K activity.
(FITC)-labeled Annexin V assay. c Caspase-3 enzymatic activity after treatment of thioridazine was determined using actyl-DEVD-7amino-4-trifluoromethyl coumarin as the substrate. d, e Caspase-3 and PARP cleavages induced by cisplatin and thioridazine treatments. Soluble protein extracts were conducted by immunoblotting for cleaved caspase- 3 and cleaved PARP. $\beta$-actin was used as an equal loading control

Finally, after treating the five cell lines (three cervical and two endometrial) with EGF ( $50 \mathrm{ng} / \mathrm{ml}, 6 \mathrm{~h}$ ), we examined the effect of thioridazine on the down-stream signaling 
Fig. 2 Effect of thioridazine on the expression of cell cycle regulatory protein and apoptosis-related genes. a Soluble protein extracts were subjected by western blot for the indicated proteins (p21, p27, cyclin A, B1, D1, CDK4, CDK2 and CDK1). $\mathbf{b}$ Expression of $\mathrm{Bcl}-2$ family genes (Bcl-2, Bcl$\mathrm{xL}, \mathrm{Bax}$ and p53) were tested by using immunoblotting. $\beta$-actin was used as loading control. c After treatment with thioridazine, transfection with p53-, p21- and Bcl-2-luciferase, respectively. After collection by centrifugation, mixed with the reaction substrate and the effect on luciferase activity were measured by using a luminometer. All experiments were performed at least three times with similar results
A
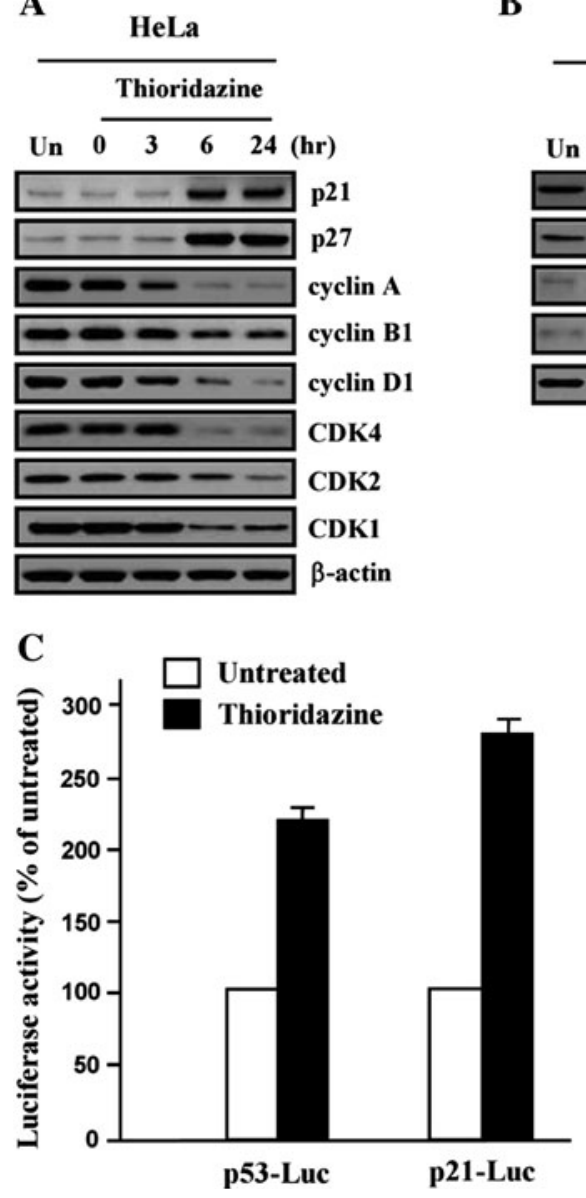

B

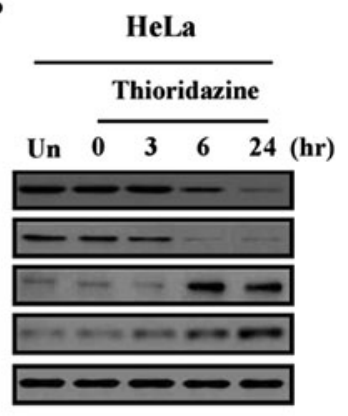

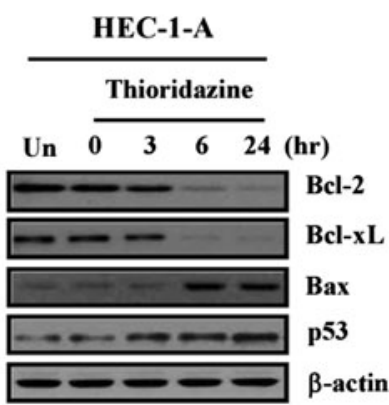

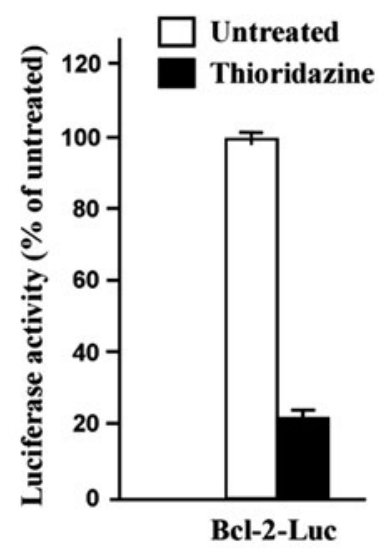

components in the PI3K/Akt pathway. Thioridazine inhibited the phosphorylation of mTOR on Ser 2448 residue and p70S6K on Thr 421 residue, a downstream target of mTOR (Fig. 4). Taken together, our results support the idea that thioridazine targets the Akt/mTOR/p70S6K signaling pathway, which leads to the inhibition of tumor growth and metastasis.

\section{Discussion}

The current study has shown that thioridazine inhibits cellular proliferation through the induction of $\mathrm{G}_{1}$ cell cycle arrest and cellular apoptosis and that the anti-proliferative effect of thioridazine on cervical and endometrial cells may result from the inhibition of $\mathrm{PI} 3 \mathrm{~K} / \mathrm{Akt} / \mathrm{mTOR} / \mathrm{p} 70 \mathrm{~S} 6 \mathrm{~K}$ signaling pathways by thioridazine, which corresponds with our previous study [26].

The PI3K/Akt/mTOR/p70S6K signaling pathways play a pivotal role in the physiological functions of human malignant tumors. PI3K modulates signaling pathways implicated in cell growth, apoptosis, or both. Regulatory factors of this pathway are frequently deregulated in an extensive number of tumor types, making it an attractive target for tumor therapy [27]. Akt activity is modulated by PI3K, which recruits Akt to the cell membrane, permitting its activation by PDK1 [28]. Akt activation induces cell cycle progression, survival, migration and metabolism through phosphorylation of various physiological factors. Activation of $\mathrm{PI} 3 \mathrm{~K}$ and Akt are reported to occur in ovarian, thyroid, breast and pancreatic tumor [29].

Based on these findings, the PI3K/Akt pathway is believed to be a promising therapeutic target for the treatment of cancer. Moreover, a body of evidence indicates that inhibition of PI3K/Akt pathway may inhibit cell growth, and increase the cytotoxic effect of conventional chemotherapeutic agents in many solid tumors [17, 30-34]. In addition, mTOR, a major down-stream target of PI3K/ Akt pathway, is well-known as an essential regulator of tumor growth and cell proliferation, protein synthesis, and the modulation of signals in various signaling pathways [27, 35].

Thus, our data suggest that thioridazine alone or with conventional cytotoxic agents may be worth to be tested in further studies as an effective therapeutic option. 

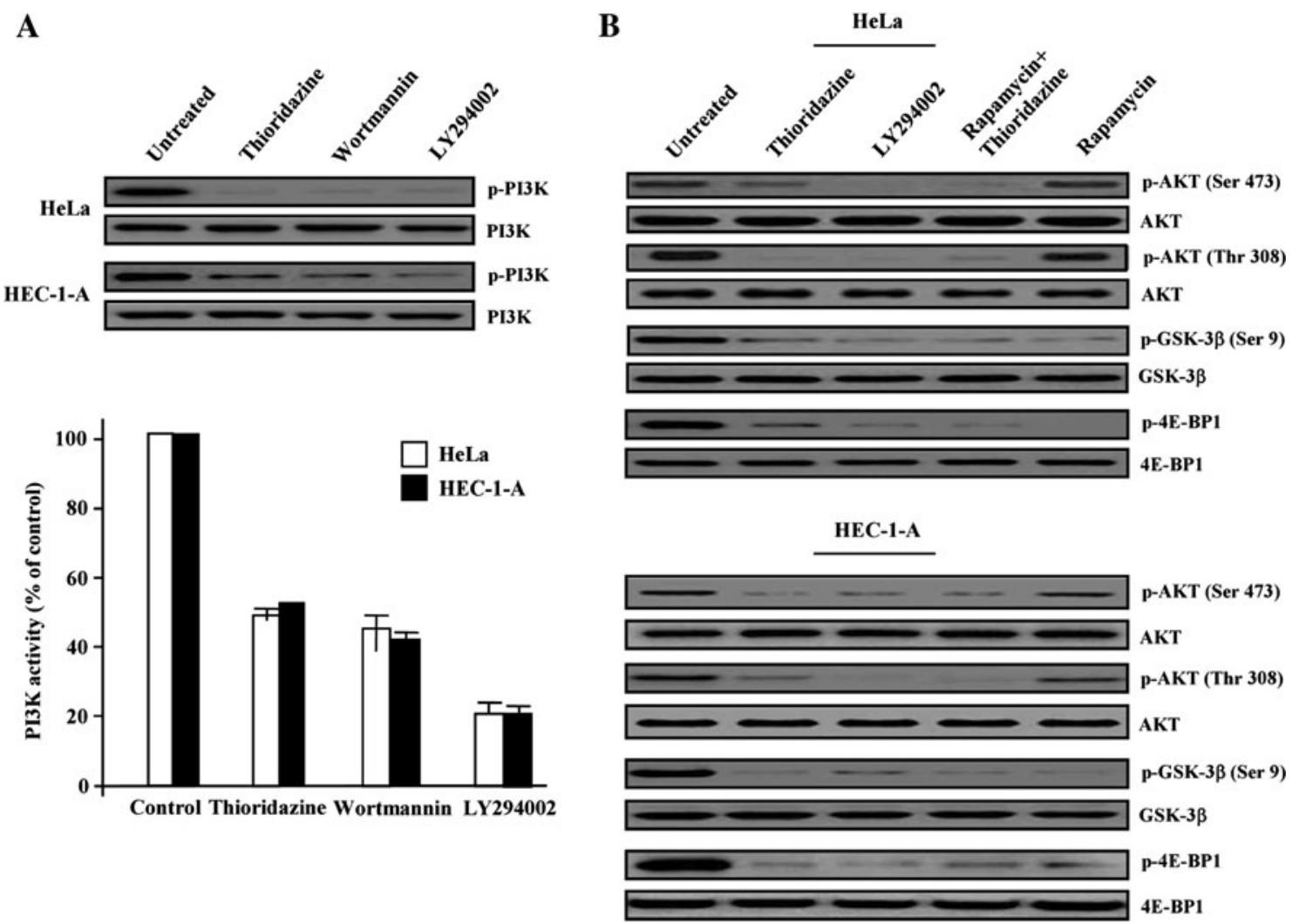

Fig. 3 Thioridazine promotes pro-apoptotic signaling through inhibition of p-Akt activation. a Effect of thioridazine and other PI3K inhibitors on PI3K activity was assayed by immunoblotting for total PI3K and phosphorylated PI3K. The reaction products were visualized using autoradiography and liquid scintillation counting. b $\mathrm{HeLa}$

and HEC-1-A cells were treated with each chemical drugs and analyzed for the indicated proteins by immunoblotting for total Akt/ phosphorylated Akt (p-Ser 473 and p-Thr 308), GSK-3 $\beta /$ phosphorylated-GSK-3 $\beta$ (p-Ser 9) and 4E-BP1/phosphorylated-4E-BP1. Results shown are representative of three independent experiments
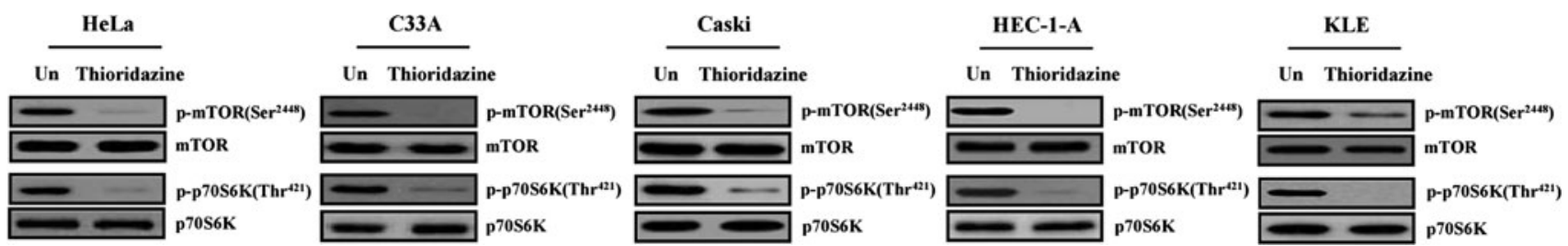

Fig. 4 Western blot analysis of down-stream components in the Akt signaling pathways with thioridazine-treated cervix cancer and endometrial cancer cells, respectively. After treatment of thioridazine, total cells lysates were prepared and investigated for phospho-

The anticancer effect of thioridazine had been demonstrated in vivo by using a mouse model system [36]. In addition, although there has been no well-designed human trial, there was a case report of successful treatment with high-dose thioridazine in a cervical cancer patient [37]. Moreover, thioridazine has already been used in cancer patients for managing depression and psychosis [38]. However, because the drug is not free from side effects, such as cardiac toxicity, movement disorder, and central nervous system effect, the toxicity of high-dose
mTOR(Ser $\left.{ }^{2448}\right)$ and phospho-p70S6K $\left(\mathrm{Thr}^{421}\right)$ protein levels by immnunoblot analysis. Unphosphorylation protein was used as an equal loading control (indicated as mTOR and p70S6K)

thioridazine should be carefully evaluated. In addition, biologically achievable dose and specificity of the drug should be determined further. Since there have been several case reports addressing the clinical efficacy of phenothiazine derivatives in cancer patients [37, 39], the clinical use of thioridazine as a targeting agent of PI3K/Akt/mTOR signaling pathway may not be unrealistic when these concerns are resolved by further research.

In summary, our data show that thioridazine can inhibit the PI3K/Akt/mTOR/p70S6K signaling pathway and exert 
cytotoxic effect on cervical and endometrial cancer cells by inducing cell cycle arrest and apoptosis. The usefulness of this agent in cancer treatment should be explored in future research.

Acknowledgments This work was supported by a grant from the National Cancer Center, Korea (NCC-0910262-3). We thank Dr. S.A. Martinis (Department of Biochemistry, University of Illinois at Urbana-Champaign, IL), and Richard Yoo (University of Washington, Seattle, WA) for critical reading of the manuscript.

Conflict of interest The authors declare that they have no conflict of interest.

Open Access This article is distributed under the terms of the Creative Commons Attribution License which permits any use, distribution, and reproduction in any medium, provided the original author(s) and the source are credited

\section{References}

1. Cowap J, Hardy J (1998) Thioridazine in the management of cancer-related sweating. J Pain Symptom Manag 15:266

2. Zhukovsky DS (2002) Fever and sweats in the patient with advanced cancer. Hematol Oncol Clin North Am 16:579-588

3. Ly KL, Chidgey J, Addington-Hall J et al (2002) Depression in palliative care: a systematic review. Part 2 treatment. Palliat Med 16:279-284

4. Ramu A, Spanier R, Rahamimoff H et al (1984) Restoration of doxorubicin responsiveness in doxorubicin-resistant $\mathrm{P} 388$ murine leukaemia cells. Br J Cancer 50:501-507

5. Akiyama S, Shiraishi N, Kuratomi Y et al (1986) Circumvention of multiple-drug resistance in human cancer cells by thioridazine, trifluoperazine, and chlorpromazine. J Natl Cancer Inst 76: 839-844

6. Munyon WH, Salo R, Briones DF (1987) Cytotoxic effects of neuroleptic drugs. Psychopharmacology 91:182-188

7. Strobl JS, Kirkwood KL, Lantz TK (1990) Inhibition of human breast cancer cell proliferation in tissue culture by the neuroleptic agents pimozide and thioridazine. Cancer Res 50:5399-5405

8. Glass-Marmor L, Morgenstern H, Beitner R (1996) Calmodulin antagonists decrease glucose 1,6-bisphosphate, fructose 1,6-bisphosphate, ATP and viability of melanoma cells. Eur J Pharmacol 313:265-271

9. Kamiwatari M, Nagata Y, Kikuchi H et al (1989) Correlation between reversing of multidrug resistance and inhibiting of $\left[{ }^{3} \mathrm{H}\right]$ azidopine photolabeling of P-glycoprotein by newly synthesized dihydropyridine analogues in a human cell line. Cancer Res 49:3190-3195

10. Pantazaki AA, Lialiaris TS (1999) A combined biochemical and cytogenetic study of thioridazine-induced damage to nucleic acids. Mutagenesis 14:243-248

11. Rodrigues T, Santos AC, Pigoso AA et al (2002) Thioridazine interacts with the membrane of mitochondria acquiring antioxidant activity toward apoptosis-potentially implicated mechanisms. Br J Pharmacol 136:136-142

12. Basta-Kaim A, Budziszewska B, Jagła G et al (2006) Inhibitory effect of antipsychotic drugs on the Con A- and LPS-induced proliferative activity of mouse splenocytes: a possible mechanism of action. J Physiol Pharmacol 57:247-264

13. Lamb J, Crawford ED, Peck D et al (2006) The connectivity map: using gene-expression signatures to connect small molecules, genes, and disease. Science 313:1929-1935
14. Franke TF, Hornik CP, Segev L et al (2003) PI3K/Akt and apoptosis: size matters. Oncogene 22:8983-8998

15. Cantley LC, Neel BG (1999) New insights into tumor suppression: PTEN suppresses tumor formation by restraining the phosphoinositide 3-kinase/AKT pathway. Proc Natl Acad Sci USA 96:4240-4245

16. Yang X, Fraser M, Moll UM et al (2006) Akt-mediated cisplatin resistance in ovarian cancer: modulation of p53 action on caspase-dependent mitochondrial death pathway. Cancer Res 66:3126-3136

17. Altomare DA, Wang HQ, Skele KL et al (2004) AKT and mTOR phosphorylation is frequently detected in ovarian cancer and can be targeted to disrupt ovarian tumor cell growth. Oncogene 23:5853-5857

18. Franke TF, Kaplan DR, Cantley LC et al (1997) Direct regulation of the Akt proto-oncogene product by phosphatidylinositol-3,4bisphosphate. Science 275:665-668

19. Vanhaesebroeck B, Waterfield MD (1999) Signaling by distinct classes of phosphoinositide 3-kinases. Exp Cell Res 253:239-254

20. Yuan ZQ, Sun N, Feldman RI et al (2000) Frequent activation of AKT2 and induction of apoptosis by inhibition of phosphoinositide-3-OH kinase/Akt pathway in human ovarian cancer. Oncogene 19:2324-2330

21. Bjornsti MA, Houghton PJ (2004) The mTOR pathways: a target for cancer therapy. Nat Rev Cancer 4:335-348

22. Bjornsti MA, Houghton PJ (2004) Lost in translation: dysregulation of cap-dependent translation and cancer. Cancer Cell 5: 519-523

23. Zhang J, Snyder SH (1992) Nitric oxide stimulates auto-ADPribosylation of glyceraldehyde-3-phosphate dehydrogenase. Proc Natl Acad Sci USA 89:9382-9385

24. Zhang J (1997) Use of biotinylated NAD to label and purify ADP-ribosylated proteins. Methods Enzymol 280:255-265

25. Fruman DA, Mauvais-Jarvis F, Pollard DA (2000) Hypoglycaemia, liver necrosis and perinatal death in mice lacking all isoforms of phosphoinositide 3-kinase p85 alpha. Nat Genet 26:379-382

26. Rho SB, Kim BR, Kang S (2011) A gene signature-based approach identifies thioridazine as an inhibitor of phosphatidylinositol-3'-kinase (PI3K)/AKT pathway in ovarian cancer cells. Gynecol Oncol 120:121-127

27. Wan X, Harkavy B, Shen N et al (2007) Rapamycin induces feedback activation of Akt signaling through an IGF-1R-dependent mechanism. Oncogene 26:1932-1940

28. Kandel ES, Hay N (1999) The regulation and activities of the multifunctional serine/threonine kinase Akt/PKB. Exp Cell Res 253:210-229

29. Blanco-Aparicio C, Renner O, Leal JF et al (2007) PTEN, more than the AKT pathway. Carcinogenesis 28:1379-1386

30. Hu L, Hofmann J, Jaffe RB (2005) Phosphatidylinositol 3-kinase mediates angiogenesis and vascular permeability associated with ovarian carcinoma. Clin Cancer Res 11:8208-8212

31. Hu L, Zaloudek C, Mills GB et al (2000) In vivo and in vitro ovarian carcinoma growth inhibition by a phosphatidylinositol 3-kinase inhibitor (LY294002). Clin Cancer Res 6:880-886

32. Mabuchi S, Altomare DA, Cheung $M$ et al (2007) RAD001 inhibits human ovarian cancer cell proliferation, enhances cisplatin-induced apoptosis, and prolongs survival in an ovarian cancer model. Clin Cancer Res 13:4261-4270

33. Mabuchi S, Kawase C, Altomare DA et al (2009) mTOR is a promising therapeutic target both in cisplatin-sensitive and cisplatin-resistant clear cell carcinoma of the ovary. Clin Cancer Res 15:5404-5413

34. Shi Y, Frankel A, Radvanyi LG et al (1995) Rapamycin enhances apoptosis and increases sensitivity to cisplatin in vitro. Cancer Res 55:1982-1988 
35. Fingar DC, Salama S, Tsou C et al (2002) Mammalian cell size is controlled by mTOR and its downstream targets S6K1 and 4EBP1/eIF4E. Genes Dev 16:1472-1487

36. Gil-Ad I, Shtaif B, Levkovitz Y et al (2006) Phenothiazines induce apoptosis in a B16 mouse melanoma cell line and attenuate in vivo melanoma tumor growth. Oncol Rep 15:107-112
37. Hercbergs A (1988) Thioridazine: a radiation enhancer in advanced cervical cancer? Lancet 2:737

38. Axelsson R, Martensson E, Alling C (1982) Serum concentration and protein binding of thioridazine and its metabolites in patients with chronic alcoholism. Eur J Clin Pharmacol 23:359-363

39. Csatary LK (1972) Chlorpromazines and cancer. Lancet 2:338-339 